Journal Afrika Statistika

Vol. 5, $\mathrm{N}^{\circ} 10,2010$, page 268-278.

$\overline{\text { Journal Afrika Statistika }}$

\title{
Extreme value theory for nonstationary random coefficients time series with regularly varying tails
}

\author{
Aliou Diop and Saliou Diouf \\ LERSTAD, Université Gaston Berger, BP. 234 Saint-Louis, Sénégal \\ Received 7 July 2010; Accepted 28 October 2010 \\ Copyright (c) 2010, Journal Afrika Statistika. All rights reserved
}

\begin{abstract}
We consider a class of nonstationary time series defined by $Y_{t}=\mu_{t}+\sum_{k=0}^{\infty} C_{t, k} \sigma_{t-k} \eta_{t-k}$ where $\left\{\eta_{t} ; t \in \mathbb{Z}\right\}$ is sequence of iid random variables with regularly varying tail probabilities, $\sigma_{t}$ is a scale parameter and $\left\{C_{t, k}, t \in\right.$ $\mathbb{Z}, k>0\}$ an infinite array of random variables identically distributed called weights. In this article, the extreme value theory of $\left\{Y_{t}\right\}$ is studied. Under mild conditions, convergence results for a point process based on the moving averages are proved.
\end{abstract}

Résumé. Nous considérons une classe de processus non stationnaires définis par $Y_{t}=\mu_{t}+\sum_{k=0}^{\infty} C_{t, k} \sigma_{t-k} \eta_{t-k}$ où $\left\{\eta_{t} ; t \in \mathbb{Z}\right\}$ est une suite de variables aléatoires indépendantes et identiquement distribuées dont les queues de distribution sont à variation régulière, $\sigma_{t}$ est un paramètre d'échelle et $\left\{C_{t, k}, t \in \mathbb{Z}, k>0\right\}$ une suite de variables de même loi appelées poids. Nous montrons que le processus de Poisson basé sur la série non stationnaire converge vaguement.

Key words: Mixing condition; Poisson process; Regular varying function; Nonstationary process.

AMS 2000 Mathematics Subject Classification : 62G32, 62G30, 62F12.

\section{Introduction}

Point processes play an important role in the study of extreme value theory of random sequences. Some extreme value data, especially in environmental contexts, often exhibit some nonstationarities. To take into account these features, it is necessary to understand the behavior of point processes based on nonstationary sequences. We quickly review the salient facts of point process theory, for notation and background of point process theory, we follow Neveu [9]; see also Kallenberg [7] and Resnick [12].

Let $\mathrm{E}$ be a state space taken to be a subset of compactified Euclidean space ( $\operatorname{such}$ as $\mathbb{R}^{d}=[-\infty ;+\infty]^{d}$ ). Let $\mathcal{E}$ be the Borel $\sigma$-algebra generated by open sets. For $x \in E$ and $A \in \mathcal{E}$, define the measure $\varepsilon_{x}$ on $\mathcal{E}$ by

$$
\varepsilon_{x}(A)= \begin{cases}1, & x \in A, \\ 0, & x \notin A .\end{cases}
$$

Let $\left\{x_{i}, i \geq 1\right\}$ be a countable collection of (not necessarily distinct) point of the space E. A point measure $m_{p}$ is defined to be a finite measure on relatively compact subsets of $\mathrm{E}$ of the form $m_{p}=\sum_{i=1}^{\infty} \varepsilon_{x_{i}}$ which is nonnegative integer-valued. The class of point measures is denoted by $M_{p}(E)$ and $\mathcal{M}_{p}(E)$ is the smallest $\sigma$-algebra making the evaluation maps $m \rightarrow m(F)$ measurable where $m \in M_{p}(E)$ and $F \in \mathcal{E}$.

Let $\mathcal{C}_{K}^{+}$be the set of all continuous, non-negative functions on the state $E$ with compact support. If $N_{n} \in M_{p}(E)$ then $N_{n}$ converges vaguely to $N\left(N_{n} \Rightarrow N\right)$ if $N_{n}(f)$ converges to $N(f)$ for every $f \in \mathcal{C}_{K}^{+}$, where $N(f)=\int f d N$. A Poisson process on $(E, \mathcal{E})$ with mean measure $\mu$ is a point process $N$ such that, for every $A \in \mathcal{E}, N(A)$ is a Poisson random variable with mean measure $\mu(A)$. If $A_{1}, \ldots, A_{k}$ are mutually independent sets then $N\left(A_{1}\right), \ldots, N\left(A_{k}\right)$ are

Aliou Diop: aliou.diop@ugb.edu.sn

Saliou Diouf: saliou_diouf@yahoo.fr 
A. Diop, S. Diouf, Journal Afrika Statistika, Vol. 5, Nº10, 2010, page 268-278.

Extreme value theory for nonstationary random coefficients time series with regularly varying tails

independent random variables. A Poisson process or a Poisson random measure with mean measure $\mu$ is denoted by $\operatorname{PRM}(\mu)$.

In this paper, we study the limit theory for extreme values of a class of nonstationary time series defined by the following relations

$$
Y_{t}=\mu_{t}+X_{t}, \quad X_{t}=\sum_{k=0}^{\infty} C_{t, k} \eta_{t-k} \sigma_{t-k}
$$

where $\left\{\eta_{t} ; t \in \mathbb{Z}\right\}$ is sequence of iid random variables with regularly varying tail probabilities, $\sigma_{t}$ is a scale parameter and $\left\{C_{t, k}, t \in \mathbb{Z}, k>0\right\}$ an infinite array of random variables identically distributed called weights.

In recent years, modeling extremes of environmental time series has been the purpose of many investigations because of its wide applicability to the analysis of phenomena such as extreme temperature, flood, storm winds and extreme ozone concentrations. See Horowitz [6] who considered the following model for daily ozone maxima $Y_{t}$ :

$$
\log \left(Y_{t}\right)=f(t)+\zeta_{t}
$$

where $f(t)$ is a deterministic part, such as a seasonal component or trend, $\left(\zeta_{t}\right)$ is a normal stationary autoregressive process. Ballerini and McCormick [1] studied the limit theory for processes of the form

$$
Y_{t}=f(t)+h(t) \zeta_{t}
$$

where $h($.$) is positive and periodic and \left\{\zeta_{t}\right\}$ is a stationary process satisfying certain mixing conditions. Niu [10] studied the limit theory for extreme values of a class of nonstationary time series with the following form

$$
Y_{t}=\mu_{t}+X_{t}, \quad X_{t}=\sum_{k=0}^{\infty} c_{k} \eta_{t-k} \sigma_{t-k}
$$

where $\sigma_{t}$ is a non random positive constant, $\left\{c_{k}\right\}$ is a sequence of real constants and $\left\{\eta_{t}\right\}$ is a sequence of iid random variables with regularly varying tail probabilities.

In this paper, we are interested in a nonstationary moving average process with random coefficients. The object of the paper is to study the extreme value theory of the nonstationary moving average process with random coefficients and appears as a direct extension of the results of Niu [10].

The rest of this paper is organized as follows. Section 2 describes the model. Section 3 contains assumptions and main results.

\section{The model}

Some extreme value data, especially in environmental contexts, often exhibit some stylized facts (see Coles, [2] and Eastoe and Tawn, 2009):

- dependence on covariate effects

- short term dependence (storms for example)

- seasonality (due to the annual cycle in meteorology)

- long-trends (due to gradual climatic changes)

- other forms of non-stationarity (switching regime motivated by interventions of central banks in finance).

To take into account these facts, we introduce a class of nonstationary time series defined by the following relations

$$
Y_{t}=\mu_{t}+X_{t}, \quad X_{t}=\sum_{k=0}^{\infty} C_{t, k} \eta_{t-k} \sigma_{t-k}
$$

We may give an example of model (3) for, say, ground-level ozone data $\left\{X_{t}\right\}$ defined by the following relation

$$
X_{t}= \begin{cases}\phi_{1} X_{t-1}+\sigma_{1 t} \eta_{t}^{(1)}, & \text { if } W_{t-\delta}>\tau, \\ \phi_{2} X_{t-1}+\sigma_{2 t} \eta_{t}^{(2)}, & \text { if } W_{t-\delta} \leq \tau,\end{cases}
$$

where $\tau$ and $\phi_{i}$ are non random constants and with threshold variable $W_{t-\delta}$. The sequences $\left\{\eta_{t}^{i}, i=1,2\right\}$ are sequence of iid random variables with regularly varying tail probabilities. 
The ground level ozone process has piecewise linear structure. It switches between two first order autoregressive process according to meteorological conditions, including daily temperature, relative humidity, wind speed and direction, which play an important role in determining the severity of ozone concentration.

In hydrological framework where the water level $X_{t}$ is observed at a given location, $W_{t-\delta}$ could be interpreted as threshold level upstream from that location and $\delta$ the delay (in terms of days, hours, for instance) for the raw wave to reach that location.

- The dependence on covariates effects can also be modeled by considering the scale parameters $\sigma_{i t}, i=1,2$ as a nonlinear function of meteorological variables of the form

$$
\sigma_{i t}=\exp \left\{\beta_{i 0}+\sum_{j=1}^{m} \beta_{i j} x_{t j}\right\} .
$$

- To allow for a seasonal component (annual for instance) in the variance, we could use

$$
\sigma_{i t}=\exp \left\{\alpha_{i 0}+\sum_{j=1}^{m} \alpha_{i j} \cos \left(\frac{2 \pi j t}{365}\right)+\beta_{i j} \sin \left(\frac{2 \pi j t}{365}\right)\right\} .
$$

- To allow for long trends due to gradual climatic changes, we could use $\mu_{t}=a_{0}+a_{1} t$.

We define $I_{1 t}=\mathbf{1}_{\left\{W_{t-\delta}>\tau\right\}}, I_{2 t}=1-I_{1 t}$. The model (4) can be written as

$$
X_{t}=\phi_{(t)} X_{t-1}+Z_{t}
$$

where

$$
\phi_{(t)}=\phi_{1} I_{1 t}+\phi_{2} I_{2 t} \quad \text { and } \quad Z_{t}=\sigma_{1 t} \eta_{t}^{(1)} I_{1 t}+\sigma_{2 t} \eta_{t}^{(2)} I_{2 t} .
$$

The equation (5) is a stochastic difference equation where the pairs $\left(\phi_{(t)}, Z_{t}\right)_{t}$ are sequences of independent and not identically distributed $\mathbb{R}^{2}$-valued random variables. The solution of $(5)$ can be written as

$$
X_{t}=\sum_{j=0}^{\infty}\left(\prod_{k=0}^{j-1} \phi_{(t-k)}\right) Z_{t-j}
$$

\section{Assumptions and Main results}

\subsection{Preliminary results}

The derivation of convergence results for point process based on non stationary sequences is far from trivial. For this, firstly we give mixing conditions and secondly we establish a general and important result for extreme value theory of non stationary process.

Let for each $n \geq 1,\left\{X_{n, i}, i \geq 1\right\}$ be a nonstationary sequence of random elements of $\mathrm{E}$.

Let $T>0$ be fixed and $\mathbf{C}$ be the finite collection of functions

$$
\mathbf{C}=\left\{h_{0}, h_{1}, \ldots, h_{m},\right\}
$$

where $h_{0}, h_{i} \in \mathcal{C}_{K}^{+}(E), h_{i} \leq 1, i=1, \ldots, m$. We say that the array $\left\{X_{n, i}, n \geq 1 i \geq 1\right\}$ satisfies the condition $D^{*}$ if for any disjoint intervals of integers $I_{1}$ and $I_{2}$ which are contained in $1,2, \ldots,[n T]$ and separated by $l$, we have

$$
\left|\mathbb{E} \prod_{j=1}^{2} \prod_{i \in I_{j}} g_{i}\left(X_{n, i}\right)-\prod_{j=1}^{2} \mathbb{E} \prod_{i \in I_{j}} g_{i}\left(X_{n, i}\right)\right| \leq \alpha_{n, l}
$$

where $\alpha_{n, l} \rightarrow 0$ as $n \rightarrow \infty, l=l(n)=o(n)$.

The condition $D^{*}$ has the following straightforward generalization. Let $I_{1}, I_{2}, \ldots, I_{k}$ be disjoint collections of integers which are separated by at least $l$ and such that $\bigcup_{j=1}^{k} I_{j} \subset[1, n T]$. 
Then

$$
\left|\mathbb{E} \prod_{j=1}^{k} \prod_{i \in I_{j}} g_{i}\left(X_{n, i}\right)-\prod_{j=1}^{k} \mathbb{E} \prod_{i \in I_{j}} g_{i}\left(X_{n, i}\right)\right| \leq(k-1) \alpha_{n, l}
$$

where $1-g_{i} \in \mathbf{C}$.

Now we can state convergence result for point processes based on nonstationary sequences under mixing conditions. This is an extension of similar theorem for independent and identically random variables and stationary processes. Our result provides the link between nonstationary processes and point process.

Theorem 1. Suppose that for each $n \geq 1,\left\{X_{n, i}, i \geq 1\right\}$ is a sequence (not necessarily stationary) of random elements of $E$ and that the array $\left\{X_{n, i}, i \geq 1, n \geq 1\right\}$ satisfies the condition $D^{*}$. Further assume that there exists a Radon measure $\nu$ on $E$ such that

$$
\sum_{j=1}^{\infty} \varepsilon_{j / n}(.) \mathbb{P}\left\{X_{n, j} \in .\right\} \rightarrow \lambda \times \nu
$$

and for any $g \in \mathcal{C}_{K}^{+}(E), g \leq 1$,

$$
\limsup _{n \rightarrow \infty} \sum_{i, l \in L_{j, k}, i \neq l} \mathbb{E} g\left(X_{n, i}\right) g\left(X_{n, l}\right)=o\left(k^{-1}\right) \quad \text { uniformly in } j
$$

where $L_{j, k}=\left\{(j-1) p_{n}+1, \ldots, j p_{n}\right\}$ and $p_{n}=\left[\frac{n}{k}\right]$. Then $N_{n}=\sum_{j=1}^{\infty} \varepsilon_{\left(j / n, X_{n, j}\right)}$ converge in $M_{p}([0, \infty) \times E)$ to $N$, where the limit is a PRM on $[0, \infty) \times E$ with mean measure $\lambda \times \nu$, where $\lambda$ is Lebesgue measure on $[0, \infty)$.

\section{Proof:}

We prove weak convergence by showing Laplace functionals converge. We follow the same lines as Davis and Resnick [3] and show that for any $f \in \mathcal{C}_{K}^{+}([0, \infty) \times E)$,

$$
\mathbb{E} \exp \left\{-\sum_{i=1}^{\infty} f\left(i / n, X_{n, i}\right)\right\} \rightarrow \exp \left\{-\int_{[0, \infty) \times E} \int\left(1-e^{-f(t, x)}\right) d t \nu(d x)\right\} .
$$

We begin to show that for $T>0$ and $f \in \mathcal{C}_{K}^{+}(E)$ that

$$
\mathbb{E} \exp \left\{-\sum_{i=1}^{[n T]} f\left(X_{n, i}\right)\right\} \rightarrow \exp \left\{-T \int_{E}\left(1-e^{-f(x)}\right) \nu(d x)\right\} .
$$

For each $n$ and $k$ fixed, we consider a partition of the integers $1,2, \ldots,[n T]$ into $2 k$ consecutive blocks of size $[[n T] / k]-$ $l(n)$ and $l(n)$, i.e.,

$$
I_{j}=\left\{(j-1) r_{n}+1, \ldots, j r_{n}-l(n)\right\} \quad I_{j}^{*}=\left\{j r_{n}-l(n)+1, \ldots, j r_{n}\right\}
$$

and

$$
I_{k}=\left\{(k-1) r_{n}+1, \ldots, k r_{n}-l(n)\right\} \quad I_{k}^{*}=\left\{k r_{n}-l(n)+1, \ldots,[n T]\right\}
$$

where $r_{n}=[[n T] / k]$. First, we split the following difference into three terms and show that each term goes to zero as $n \rightarrow \infty$ :

$$
\begin{aligned}
& \left|\mathbb{E} \exp \left\{-\sum_{i=1}^{[n T]} f\left(X_{n, i}\right)\right\}-\prod_{j=1}^{k} \mathbb{E} \exp \left\{-\sum_{i \in I_{j} \cup I_{j}^{*}} f\left(X_{n, i}\right)\right\}\right| \\
& \leq\left|\mathbb{E} \exp \left\{-\sum_{i=1}^{[n T]} f\left(X_{n, i}\right)\right\}-\mathbb{E} \exp \left\{-\sum_{j=1}^{k} \sum_{i \in I_{j}} f\left(X_{n, i}\right)\right\}\right| \\
& +\left|\mathbb{E} \exp \left\{-\sum_{j=1}^{k} \sum_{i \in I_{j}} f\left(X_{n, i}\right)\right\}-\prod_{j=1}^{k} \mathbb{E} \exp \left\{-\sum_{i \in I_{j}} f\left(X_{n, i}\right)\right\}\right| \\
& +\left|\prod_{j=1}^{k} \mathbb{E} \exp \left\{-\sum_{i \in I_{j}} f\left(X_{n, i}\right)\right\}-\prod_{j=1}^{k} \mathbb{E} \exp \left\{-\sum_{i \in I_{j} \cup I_{j}^{*}} f\left(X_{n, i}\right)\right\}\right|
\end{aligned}
$$




\section{Step 1:}

$$
=A_{1}+A_{2}+A_{3}
$$

Using the mixing condition, we have $A_{2} \leq(k-1) \alpha_{n, l}$. Then $A_{2}$ goes to zero as $n \rightarrow \infty$.

\section{Step 2:}

$$
\begin{aligned}
A_{1} & =\left|\mathbb{E} \exp \left\{-\sum_{i=1}^{[n T]} f\left(X_{n, i}\right)\right\}-\mathbb{E} \exp \left\{-\sum_{j=1}^{k} \sum_{i \in I_{j}} f\left(X_{n, i}\right)\right\}\right| \\
& =\left|\mathbb{E}\left(\prod_{i=1}^{[n T]} \exp -f\left(X_{n, i}\right)-\prod_{j=1}^{k} \prod_{i \in I_{j}} \exp -f\left(X_{n, i}\right)\right)\right|
\end{aligned}
$$

Note that $\{1,2, \ldots,[n T]\}=\bigcup_{j=1}^{k} I_{j} \cup I_{j}^{*}$. Using the following inequality

$$
\left|\prod_{i=1}^{n} x_{i}-\prod_{i=1}^{n} y_{i}\right| \leq \sum_{i=1}^{n}\left|x_{i}-y_{i}\right|, \quad 0 \leq x_{i}, y_{i} \leq 1 \quad i=1, \ldots, n
$$

we have

$$
\begin{aligned}
A_{1} & \leq \mathbb{E} \sum_{j=1}^{k}\left(1-\exp \left\{-\sum_{i \in I_{j}^{*}} f\left(X_{n, i}\right)\right\}\right) \\
& \leq \sum_{j=1}^{k} \sum_{i \in I_{j}^{*}} \int\left(1-e^{-f(x)}\right) \mathbb{P}\left(X_{n, i} \in d x\right) \\
& \leq \sum_{j=1}^{k} \int\left(1-e^{-f(x)}\right) \sum_{i \in I_{j}^{*}} \mathbb{P}\left(X_{n, i} \in d x\right) \\
& \leq \sum_{j=1}^{k} \int\left(1-e^{-f(x)}\right) \sum_{i=1}^{\infty} \varepsilon_{i / n}\left(\frac{j T}{k}-c, \frac{j T}{k}\right) \mathbb{P}\left(X_{n, i} \in d x\right)
\end{aligned}
$$

for all $0<c<1$ since $l(n)=o(n)$. Thus by $(7)$

$$
A_{1} \rightarrow c k \int\left(1-e^{-f(x)}\right) \nu(d x) \quad \text { for all } 0<c<1
$$

Therefore $A_{1}$ converges to zero as $n \rightarrow \infty$.

\section{Step 3:}

$$
\begin{aligned}
A_{3} & =\left|\prod_{j=1}^{k} \mathbb{E} \exp \left\{-\sum_{i \in I_{j}} f\left(X_{n, i}\right)\right\}-\prod_{j=1}^{k} \mathbb{E} \exp \left\{-\sum_{i \in I_{j} \cup I_{j}^{*}} f\left(X_{n, i}\right)\right\}\right| \\
& \leq \sum_{j=1}^{k}\left|\mathbb{E} \exp \left\{-\sum_{i \in I_{j}} f\left(X_{n, i}\right)\right\}-\mathbb{E} \exp \left\{-\sum_{i \in I_{j} \cup I_{j}^{*}} f\left(X_{n, i}\right)\right\}\right| \\
& \leq \sum_{j=1}^{k} \mathbb{E}\left(1-\prod_{i \in I_{j}^{*}} e^{-f\left(X_{n, i}\right)}\right) \\
& \leq \sum_{j=1}^{k} \sum_{i \in I_{j}^{*}} \mathbb{E}\left(1-e^{-f\left(X_{n, i}\right)}\right) .
\end{aligned}
$$

Using the same arguments as in Step 2, the right bound of the above inequality goes to zero as $n \rightarrow \infty$.

\section{Step 4:}

Now let us show that

$$
\prod_{j=1}^{k} \mathbb{E} \exp \left\{-\sum_{i \in I_{j} \cup I_{j}^{*}} f\left(X_{n, i}\right)\right\} \rightarrow \exp \left\{-T \int_{E}\left(1-e^{-f(x)}\right) \nu(d x)\right\} .
$$


Set $y_{i}=1-e^{-f\left(X_{n, i}\right)}$ and apply the following inequality

$$
1-\sum_{i \in I_{j} \cup I_{j}^{*}} y_{i} \leq \prod_{i \in I_{j} \cup I_{j}^{*}}\left(1-y_{i}\right) \leq 1-\sum_{i \in I_{j} \cup I_{j}^{*}} y_{i}+\sum_{i<l \in I_{j} \cup I_{j}^{*}} y_{i} y_{l} \quad 0 \leq y_{i} \leq 1 .
$$

We have

$$
\begin{gathered}
1-\sum_{i \in I_{j} \cup I_{j}^{*}}\left(1-e^{-f\left(X_{n, i}\right)}\right) \leq \prod_{i \in I_{j} \cup I_{j}^{*}}\left(e^{-f\left(X_{n, i}\right)}\right) \leq 1-\sum_{i \in I_{j} \cup I_{j}^{*}}\left(1-e^{-f\left(X_{n, i}\right)}\right) \\
+\sum_{i<l \in I_{j} \cup I_{j}^{*}}\left(1-e^{-f\left(X_{n, i}\right)}\right)\left(1-e^{-f\left(X_{n, l}\right)}\right) .
\end{gathered}
$$

We first show that

$$
\mathbb{E} \sum_{i \in I_{j} \cup I_{j}^{*}}\left(1-e^{-f\left(X_{n, i}\right)}\right)
$$

has the same limit as

$$
\int\left(1-e^{-f(x)}\right) \sum_{i=1}^{\infty} \varepsilon_{i / n}\left[\frac{(j-1) T}{k}, \frac{j T}{k}\right) \mathbb{P}\left(X_{n, i} \in d x\right) .
$$

It suffices to prove

$$
B_{1}=\sum_{i=1}^{\infty} \varepsilon_{i / n}\left[\frac{(j-1) T}{k}, \frac{(j-1) r_{n}}{n}+\frac{1}{n}\right) \mathbb{P}\left(X_{n, i} \in d x\right)
$$

and

$$
B_{2}=\sum_{i=1}^{\infty} \varepsilon_{i / n}\left[\frac{j r_{n}}{n}, \frac{j T}{k}\right) \mathbb{P}\left(X_{n, i} \in d x\right)
$$

go to zero. Indeed for all $\gamma_{1}>0$ there exists $N_{1}$ such that for all $n \geq N_{1},\left|\frac{(j-1) r_{n}}{n}+\frac{1}{n}-\frac{(j-1) T}{k}\right| \leq \gamma_{1}$. Then

$$
B_{1} \leq \sum_{i=1}^{\infty} \varepsilon_{i / n}\left[\frac{(j-1) T}{k}, \frac{(j-1) T}{k}+\gamma_{1}\right) \mathbb{P}\left(X_{n, i} \in d x\right) \rightarrow \gamma_{1} \nu(d x)
$$

Using the same argument, we have for all $\gamma_{2}>0$ there exists $N_{2}$ such that for all $n \geq N_{2}, B_{2} \leq \gamma_{2} \nu(d x)$. Hence $B_{1}$ and $B_{2}$ tend to zero as $n \rightarrow \infty$. We can conclude that

$$
\mathbb{E} \sum_{i \in I_{j} \cup I_{j}^{*}}\left(1-e^{-f\left(X_{n, i}\right)}\right) \rightarrow \frac{T}{k} \int\left(1-e^{-f(x)}\right) \nu(d x)
$$

Secondly, from (7) and (8), we have

$$
\begin{aligned}
& \mathbb{E} \sum_{i<l \in I_{j} \cup I_{j}^{*}}\left(1-e^{-f\left(X_{n, i}\right)}\right)\left(1-e^{-f\left(X_{n, l}\right)}\right) \\
& =\sum_{\substack{i<l \in I_{j} \cup I_{j}^{*} \\
\rightarrow 0 .}} \mathbb{E}\left(1-e^{-f\left(X_{n, i}\right)}\right)\left(1-e^{-f\left(X_{n, l}\right)}\right) \\
&
\end{aligned}
$$

Combining (12) and (13), we get (11).

Following the same lines as in Davis and Resnick [3], let $f \in \mathcal{C}_{K}^{+}([0, \infty))$ and suppose that the support of $f$ is contained in $[0, T] \times K, K$ is a subset of $E$ with $\nu(\partial K)$. Given $\varepsilon>0$, there exists a partition of $[0, T]$

$$
0=a_{1}<b_{1}<a_{2}<b_{2}<\ldots<a_{m}<b_{m}=T
$$

such that

$$
\sum_{j=1}^{m-1}\left(a_{j+1}-b_{j}\right) \leq \varepsilon / \nu(K)
$$

and

$$
\sup _{t \in\left(a_{j}, b_{j}\right], x \in E}\left|f\left(b_{j}, x\right)-f(t, x)\right|<\varepsilon T^{-1} / \nu(K), \quad j=1, \ldots, m
$$


A. Diop, S. Diouf, Journal Afrika Statistika, Vol. 5, Nº10, 2010, page 268-278.

Now, we split the following difference into four terms and show that each term goes to zero as $n \rightarrow \infty$ :

$$
\begin{aligned}
& \left|\mathbb{E} \exp \left(-\sum_{i=1}^{\infty} f\left(\frac{i}{n}, X_{n, i}\right)\right)-\exp \left\{-\int_{[0, \infty) \times E} \int\left(1-e^{-f(t, x)}\right) d t \nu(d x)\right\}\right| \\
& \leq\left|\mathbb{E} \exp \left(-\sum_{i=1}^{\infty} f\left(\frac{i}{n}, X_{n, i}\right)\right)-\mathbb{E} \exp \left\{-\sum_{j=1}^{m} \sum_{i=1}^{\infty} \varepsilon_{\frac{i}{n}}\left(a_{j}, b_{j}\right] f\left(\frac{i}{n}, X_{n, i}\right)\right\}\right| \\
& +\left|\mathbb{E} \exp \left\{-\sum_{j=1}^{m} \sum_{i=1}^{\infty} \varepsilon_{\frac{i}{n}}\left(a_{j}, b_{j}\right] f\left(\frac{i}{n}, X_{n, i}\right)\right\}-\mathbb{E} \exp \left\{-\sum_{j=1}^{m} \sum_{i=1}^{\infty} \varepsilon_{\frac{i}{n}}\left(a_{j}, b_{j}\right] f\left(b_{j}, X_{n, i}\right)\right\}\right| \\
& +\left|\mathbb{E} \exp \left\{-\sum_{j=1}^{m} \sum_{i=1}^{\infty} \varepsilon_{\frac{i}{n}}\left(a_{j}, b_{j}\right] f\left(b_{j}, X_{n, i}\right)\right\}-\prod_{j=1}^{m} \mathbb{E} \exp \left\{-\sum_{i=1}^{\infty} \varepsilon_{\frac{i}{n}}\left(a_{j}, b_{j}\right] f\left(b_{j}, X_{n, i}\right)\right\}\right| \\
& +\left|\prod_{j=1}^{m} \mathbb{E} \exp \left\{-\sum_{i=1}^{\infty} \varepsilon_{\frac{i}{n}}\left(a_{j}, b_{j}\right] f\left(b_{j}, X_{n, i}\right)\right\}-\exp \left\{-\int_{[0, \infty) \times E}\left(1-e^{-f(t, x)}\right) d t \nu(d x)\right\}\right| \\
& =C_{1}+C_{2}+C_{3}+C_{4} .
\end{aligned}
$$

First,

$$
\begin{gathered}
C_{1} \leq \mathbb{E}\left[1-\exp \left(1-\exp \left\{\sum_{j=1}^{m} \sum_{i=1}^{\infty} \varepsilon_{\frac{i}{n}}\left(a_{j}, b_{j}\right] f\left(\frac{i}{n}, X_{n, i}\right)\right\}\right)\right] \\
\leq \mathbb{P}\left(\bigcup_{j=1}^{m-1} \bigcup_{\frac{i}{n} \in\left(b_{j}, a_{j+1}\right]} X_{n, i} \in K\right) \\
\leq \sum_{j=1}^{m-1} \sum_{i=1}^{\infty} \varepsilon_{\frac{i}{n}}\left(b_{j}, a_{j+1}\right] \mathbb{P}\left\{X_{n, i} \in K\right\}
\end{gathered}
$$

By (7), this last bound goes to $\sum_{j=1}^{m-1}\left(a_{j+1}-b_{j}\right) \nu(K) \leq \varepsilon$.

$$
\begin{aligned}
C_{2} & \leq \sum_{j=1}^{m} \sum_{i=1}^{\infty} \varepsilon_{\frac{i}{n}}\left(a_{j}, b_{j}\right] \mathbb{E}\left(\left|f\left(\frac{i}{n}, X_{n, i}\right)-f\left(b_{j}, X_{n, i}\right)\right| \mathbb{I}_{X_{n, i} \in K}\right) \\
& \leq \frac{\varepsilon}{T \nu(K)} \sum_{j=1}^{m} \sum_{i=1}^{\infty} \varepsilon_{\frac{i}{n}}\left(a_{j}, b_{j}\right] \mathbb{P}\left\{X_{n, i} \in K\right\} .
\end{aligned}
$$

This last term tends to $\varepsilon T^{-1} \sum_{j=1}^{m}\left(b_{j}-a_{j}\right) \leq \varepsilon$. It is easy, using the mixing condition, that $C_{3} \leq(m-1) \alpha_{n, l(n)}$.

We show that $C_{4}$ goes to zero by combining Lemma 1 below and the same arguments as in Davis and Resnick [3].

Lemma 1. For all $j$

$$
\mathbb{E} \exp \left\{-\sum_{i=1}^{\infty} \varepsilon_{\frac{i}{n}}\left(a_{j}, b_{j}\right] f\left(b_{j}, X_{n, i}\right)\right\} \rightarrow \exp \left\{-\left(b_{j}-a_{j}\right) \int_{E}\left(1-e^{-f\left(b_{j}, x\right)}\right) \nu(d x)\right\} .
$$

\section{Proof :}

The proof is similar to the proof of (10). Il suffices to split the interval $\left[\left[n a_{i}\right],\left[n b_{i}\right]\right]$ into $2 k$ - consecutive blocks

$$
I_{j}=\left\{\left[n a_{i}\right]+(j-1) r_{n}, \ldots,\left[n a_{i}\right]+j r_{n}-l(n)-1\right\} \quad I_{j}^{*}=\left\{\left[n a_{i}\right]+j r_{n}-l(n), \ldots,\left[n a_{i}\right]+j r_{n}-1\right\}
$$

for $j=1, \ldots, k$ where $r_{n}=\left(\left[n b_{i}\right]-\left[n a_{i}\right]+1\right) / k$. Note that we do not need the condition of stationarity as in Davis and Resnick [3]. 
A. Diop, S. Diouf, Journal Afrika Statistika, Vol. 5, Nº10, 2010, page 268-278.

Extreme value theory for nonstationary random coefficients time series with regularly varying tails

\subsection{Main results}

To study the limit theorem for point processes based on the nonstationary time series (3), we will use Theorem 1 and the following assumptions. We assume that the absolute value of each weight $C_{t, k}$ has an upper endpoint $c_{k}$ defined by

$$
c_{k}=\sup \left\{c: \mathbb{P}\left(\left|C_{t, k}\right| \leq c\right)<1\right\}, \quad k=1,2, \ldots
$$

Assume the following conditions hold:

$\mathbf{H}_{\mathbf{1}}$ - The sequence of random variables $\left\{\eta_{t}, t \in \mathbb{Z}\right\}$ is a sequence of independent, identically distributed (i.i.d) random variables and satisfy the condition of regularly varying tail probabilities

$$
\mathbb{P}\left(\left|\eta_{1}\right|>x\right)=x^{-\alpha} L(x),
$$

where $\alpha>0$ and $\mathrm{L}$ is a slowly varying function at infinity that is $\lim _{t \rightarrow \infty} \frac{L(t x)}{L(t)}=1, \forall x>0$ and tail balancing condition,

$$
\lim _{x \rightarrow \infty} \frac{\mathbb{P}\left(\eta_{1}>x\right)}{\mathbb{P}\left(\left|\eta_{1}\right|>x\right)}=\pi_{0}, \quad \lim _{x \rightarrow \infty} \frac{\mathbb{P}\left(\eta_{1}<-x\right)}{\mathbb{P}\left(\left|\eta_{1}\right|>x\right)}=1-\pi_{0}
$$

where $0<\pi_{0} \leq 1$. Let $a_{n}$ be the $1-n^{-1}$ quantile of $\left|\eta_{1}\right|$ :

$$
a_{n}=\inf \left\{x: \mathbb{P}\left(\left|\eta_{1}\right| \leq x\right) \geq 1-n^{-1}\right\}
$$

The condition of regularly varying tail probabilities satisfied by the sequence of random variables $\left\{\eta_{t}, t \in \mathbb{Z}\right\}$ is equivalent to vague convergence

$$
n \mathbb{P}\left(a_{n}^{-1} \eta_{1} \in .\right) \rightarrow \nu(.),
$$

where $\nu$ has density $\nu(d x)=\alpha \pi_{0} x^{-\alpha-1} d x \mathbb{I}_{(0, \infty]}(x)+\alpha\left(1-\pi_{0}\right)(-x)^{-\alpha-1} d x \mathbb{I}_{[-\infty, 0)}(x)$.

$\mathbf{H}_{\mathbf{2}}$ - The array $\left\{C_{t, k}, t \in \mathbb{Z}, k \geq 0\right\}$ is independent of $\left\{\eta_{t}, t \in \mathbb{Z}\right\}$.

$\mathbf{H}_{\mathbf{3}}-$ For each fixed $m$, the sequence $\left\{\left(C_{t, 0}, \ldots, C_{t, m}\right), t \in \mathbb{Z}\right\}$ is strongly mixing.

$\mathbf{H}_{4}-$ For some $\delta>0 \quad \sum_{k=1}^{\infty} c_{k}^{1-\delta}<\infty \quad$ and $\quad \sum_{k=1}^{\infty} \sigma_{t-k}^{\alpha} c_{k}^{\delta \alpha}<\infty$.

Furthermore we assume that for fixed $k \geq 0$,

$$
\frac{1}{n} \sum_{j=1}^{n} \sigma_{j-k}^{\alpha} \rightarrow \sigma_{-k}^{\alpha}, \quad \text { as } n \rightarrow \infty
$$

where $\sigma_{-k}>0$. When $k=0$, this assumption is required by Niu [10].

Now let

$$
A_{n, t}^{(m)}=\left(a_{n}^{-1}\left(\sigma_{t} \eta_{t}, \ldots, \sigma_{t-m} \eta_{t-m}\right),\left(C_{t, 0}, \ldots, C_{t, m}\right)\right)
$$

and assume that the $\mathbb{R}^{\infty}$-valued random elements $\mathbf{C}_{t}=\left\{C_{t, k}, k \geq 0\right\}$ form the stationary sequence $\left\{\mathbf{C}_{t}, t \geq 1\right\}$. Assume the $\mathbb{R}^{\infty}$-valued random elements $V_{t}=\left(V_{t, 0}, V_{t, 1}, \ldots\right), t \in \mathbb{Z}$ has the same distribution as $\mathbf{C}_{0}$.

It is known from Niu [10] that for the process $\left(X_{t}\right)$ defined in $(1)$,

$$
N_{n}=\sum_{t=1}^{n} \varepsilon_{a_{n}^{-1} X_{t}} \Rightarrow \sum_{t=1}^{\infty} \sum_{k=1}^{\infty} \varepsilon_{c_{k} j_{t}}
$$

in $M_{p}([-\infty, \infty] \backslash\{0\})$ where $\sum_{t=1}^{\infty} \varepsilon_{j_{t}}$ is a PRM with density

$$
\mu(d x)=\sigma^{\alpha}\left(\pi_{0} \alpha x^{-\alpha-1} d x \mathbb{I}_{(0, \infty]}(x)+\left(1-\pi_{0}\right) \alpha(-x)^{-\alpha-1} d x \mathbb{I}_{[-\infty, 0)}(x)\right) .
$$

The main result of this section is formalized through the following theorem, which discusses the weak convergence of the sequence of point processes based on $\left(a_{n}^{-1} X_{k}\right)_{k \in \mathbb{N}}$ to a function of a PRM.

Theorem 2. Suppose that the non stationary sequence $\left(X_{t}\right)$ is given by (3). Assume that the conditions $H_{1}-H_{4}$ hold. Then we have this convergence in $M_{p}([-\infty, \infty] \backslash\{0\})$

$$
\sum_{t=1}^{n} \varepsilon_{a_{n}^{-1} X_{t}} \Rightarrow \sum_{t=1}^{\infty} \sum_{k=1}^{\infty} \varepsilon_{j_{t} V_{t, k}}
$$




\section{Proof}

The random vectors $A_{n, t}^{(m)}$ defined in (20) have the following properties:

- The sequence $\left\{A_{n, t}^{(m)}, t \geq 1\right\}$ satisfies the mixing condition $D^{*}$, by $\mathbf{H}_{\mathbf{2}}$ and $\mathbf{H}_{\mathbf{3}}$.

- For each $m$, there exists a Radon measure $\mu_{m}$ on the product space $E^{2 m+2}=([0, \infty) \times[-\infty, \infty] \backslash\{0\})^{2 m+2}$ such that

$$
\sum_{j=1}^{\infty} \varepsilon_{j / n}(.) \mathbb{P}\left\{A_{n, j}^{(m)} \in .\right\} \rightarrow \lambda \times \mu_{m} .
$$

It suffices to show that for any $b>0$

$$
\sum_{j=1}^{\infty} \varepsilon_{j / n}([0, b)) \mathbb{P}\left\{A_{n, j}^{(m)} \in .\right\} \rightarrow b \mu_{m}(.) .
$$

Notice that by $\mathbf{H}_{\mathbf{2}}$ and the definition of $a_{n}$ given in (17), we have

$$
\begin{aligned}
& \sum_{j=1}^{\infty} \varepsilon_{j / n}([0, b)) \mathbb{P}\left\{A_{n, j}^{(m)} \in\left(\left(d z_{0}, \ldots, d z_{m}\right),\left(d x_{0}, \ldots, d x_{m}\right)\right)\right\} \\
= & \sum_{j=1}^{[n b]} \mathbb{P}\left\{a_{n}^{-1}\left(\sigma_{j} \eta_{j}, \ldots, \sigma_{j-m} \eta_{j-m}\right) \in\left(d z_{0}, \ldots, d z_{m}\right),\left(C_{j, 0}, \ldots, C_{j, m}\right) \in\left(d x_{0}, \ldots, d x_{m}\right)\right\} \\
= & \sum_{j=1}^{[n b]} \mathbb{P}\left\{a_{n}^{-1}\left(\sigma_{j} \eta_{j}, \ldots, \sigma_{j-m} \eta_{j-m}\right) \in\left(d z_{0}, \ldots, d z_{m}\right)\right\} \times \mathbb{P}\left\{\left(C_{j, 0}, \ldots, C_{j, m}\right) \in\left(d x_{0}, \ldots, d x_{m}\right)\right\}
\end{aligned}
$$

where $[n b]$ denotes the integer part of $n b$. This last term has the same limit as

$$
\frac{1}{n} \sum_{j=1}^{[n b]} \sum_{k=0}^{m} \sigma_{j-k}^{\alpha} \nu\left(d z_{k}\right) \prod_{l \neq k}^{m} \delta_{0}\left(d z_{l}\right) F_{m}\left(d x_{0}, \ldots, d x_{m}\right)
$$

which converges to

$$
b \sum_{k=0}^{m} \sigma_{-k}^{\alpha} \nu\left(d z_{k}\right) \prod_{l \neq k} \delta_{0}\left(d z_{l}\right) F_{m}\left(d x_{0}, \ldots, d x_{m}\right)
$$

by (19), where $F_{m}$ is the distribution function of $\left\{C_{t, 0}, \ldots, C_{t, m}\right\}$.

- For all $g \in \mathcal{C}_{K}^{+}\left(E_{m+1} \times[-\infty, \infty]^{m+1}\right)$, we have

$$
\lim _{m \rightarrow \infty} \limsup _{n \rightarrow \infty} \sum_{i, l \in L_{j, m}, i \neq l} \mathbb{E} g\left(A_{n, i}^{(m)}\right) g\left(A_{n, l}^{(m)}\right)=0
$$

where $L_{j, m}=\left\{(j-1) p_{n}+1, \ldots, j p_{n}\right\}$ and $p_{n}=\left[\frac{n}{m}\right]$. Actually, let $K$ a compact subset of $E_{1}$ and assume that $g$ has compact support contained in $K_{1}=(K \times[-\infty, \infty])^{m+1}$

$$
\mathbb{E} g\left(A_{n, i}^{(m)}\right) g\left(A_{n, l}^{(m)}\right) \leq \mathbb{P}\left(a_{n}^{-1} \sigma_{i} \eta_{i} \in K, a_{n}^{-1} \sigma_{l} \eta_{l} \in K\right) .
$$

Since $\eta_{i}$ and $\eta_{l}$ are independent for all $i \neq l$, we have

$$
\sum_{i, l \in L_{j, m}, i \neq l} \mathbb{E} g\left(A_{n, i}^{(m)}\right) g\left(A_{n, l}^{(m)}\right) \leq \sum_{i \in L_{j, m}} \mathbb{P}\left(a_{n}^{-1} \sigma_{i} \eta_{i} \in K\right) \sum_{l \in L_{j, m}} \mathbb{P}\left(a_{n}^{-1} \sigma_{l} \eta_{l} \in K\right) .
$$

Using the same arguments as in the proof of Theorem 1, Step 4, it is easy to see that

$$
\sum_{i \in L_{j, m}} \mathbb{P}\left(a_{n}^{-1} \sigma_{i} \eta_{i} \in K\right)
$$

has the same limit as

$$
\sum_{i=1}^{\infty} \varepsilon_{i / n}\left[\frac{(j-1)}{m}, \frac{j}{m}\right) \mathbb{P}\left(a_{n}^{-1} \sigma_{i} \eta_{i} \in K\right) .
$$

This last term tends to $\frac{1}{m} \mu(K)$, by Lemma 2.2 of Niu [10]. Therefore, (22) follows. 
A. Diop, S. Diouf, Journal Afrika Statistika, Vol. 5, Nº10, 2010, page 268-278.

Now we can apply Theorem 1 for the sequence $\left\{A_{n, t}^{(m)}, t \geq 1\right\}$

$$
\sum_{t=1}^{n} \varepsilon_{\left(A_{n, t}^{(m)}\right)} \Rightarrow \sum_{t=1}^{\infty} \sum_{k=0}^{m} \varepsilon_{\left(j_{t} \mathbf{e}_{k}, V_{t, 0}, \ldots, V_{t, m}\right)}
$$

in $M_{p}\left(E_{m+1} \times[-\infty, \infty]^{m+1}\right)$ where $E_{m+1}=[-\infty, \infty]^{m+1} \backslash\{\mathbf{0}\}$.

Now let $\mathbf{V}_{\mathbf{t}}=\left(C_{t, 0} Z_{t}, \ldots, C_{t, m} Z_{t-m}\right)$ and

$$
g_{i, m}\left(x_{0}, \ldots, x_{m}, u_{0}, \ldots, u_{m}\right)=\left\{\begin{array}{c}
x_{i} u_{i}, \text { if } u_{i}<\infty, \\
0, \text { otherwise, }
\end{array}\right.
$$

$g_{i, m}$ is a continuous mapping from $E_{m+1} \times[-\infty, \infty]^{m+1}$ into $[-\infty, \infty] \backslash\{0\}$. By Proposition 3.2 of Davis and Resnick [4], this induces a continuous mapping from $M_{p}\left(E_{m+1} \times[-\infty, \infty]^{m+1}\right)$ into $M_{p}([-\infty, \infty] \backslash\{0\})$. Thus from $(23)$ and the continuous mapping Theorem, we get

$$
\sum_{t=1}^{n} \varepsilon_{a_{n}^{-1} \mathbf{V}_{\mathbf{t}}} \Rightarrow \sum_{t=1}^{\infty} \sum_{k=0}^{m} \varepsilon_{j_{t} V_{t, k} \mathbf{e}_{k}} .
$$

An application of the continuous mapping Theorem gives

$$
\sum_{t=1}^{n} \varepsilon_{a_{n}^{-1} \sum_{k=0}^{m} C_{t, k} Z_{t-k}} \Rightarrow \sum_{t=1}^{\infty} \sum_{k=0}^{m} \varepsilon_{j_{t} V_{t, k}} .
$$

Recall that $X_{t}=\sum_{k=0}^{\infty} C_{t, k} Z_{t-k}$. To establish (21), it suffices to show that

$$
\lim _{m \rightarrow \infty} \limsup _{n \rightarrow \infty} \mathbb{P}\left\{\left|\sum_{t=1}^{n} f\left(a_{n}^{-1} \sum_{k=0}^{m} C_{t, k} Z_{t-k}\right)-\sum_{i=1}^{n} f\left(a_{n}^{-1} X_{t}\right)\right|>\zeta\right\}=0
$$

for all $\zeta>0$ and $f \in \mathcal{C}_{K}^{+}\left(E_{1}\right)$ (Resnick, 1987). Taking into account the support of $f$, for some $\theta>0$, this last probability is bounded by

$$
\begin{aligned}
\mathbb{P}\left[\left|\sum_{t=0}^{n} a_{n}^{-1}\left(\sum_{k=0}^{m} C_{t, k} Z_{t-k}\right)-\sum_{t=0}^{n} a_{n}^{-1}\left(\sum_{k=0}^{\infty} C_{t, k} Z_{t-k}\right)\right|>\theta\right] & \leq \mathbb{P}\left[\left|\sum_{t=0}^{n} \sum_{k=m+1}^{\infty} a_{n}^{-1}\left(C_{t, k} Z_{t-k}\right)\right|>\theta\right] \\
& \leq \mathbb{P}\left[\sum_{t=0}^{n} \sum_{k=m+1}^{\infty}\left|C_{t, k}\right|\left|a_{n}^{-1} \sigma_{t-k} \eta_{t-k}\right|>\theta\right] \\
& \leq \mathbb{P}\left[\sum_{t=0}^{n} \sum_{k=m+1}^{\infty} c_{k}\left|a_{n}^{-1} \sigma_{t-k} \eta_{t-k}\right|>\theta\right] \\
& \leq \mathbb{P}\left[\sum_{t=0}^{n} \sum_{k=m+1}^{\infty} c_{k}\left|a_{n}^{-1} \sigma_{t-k} \eta_{t-k}\right|>\sum_{k=m+1}^{\infty} c_{k}^{1-\delta} \theta\right] \\
& \leq \sum_{k=m+1}^{\infty} n \mathbb{P}\left[\left|a_{n}^{-1} \sigma_{t-k} \eta_{t-k}\right|>c_{k}^{-\delta} \theta\right] .
\end{aligned}
$$

By (18), (16) and $H_{4}$, we obtain

$$
\begin{aligned}
n \mathbb{P}\left[a_{n}^{-1}\left|\sigma_{t-k} \eta_{k}\right|>c_{k}^{-\delta} \theta\right] & =n \mathbb{P}\left[\left|\eta_{k}\right|>a_{n} \theta \sigma_{t-k}^{-1} c_{k}^{-\delta}\right] \\
& \rightarrow \pi_{0}^{-1}\left(\theta\left|\sigma_{t-k}\right|^{-1} c_{k}^{-\delta}\right)^{-\alpha} .
\end{aligned}
$$

Then

$$
\lim _{n \rightarrow \infty} \lim _{m \rightarrow \infty} \sum_{k=m+1}^{\infty} n \mathbb{P}\left[\left|a_{n}^{-1} \sigma_{t-k} \eta_{t-k}\right|>c_{k}^{-\delta} \theta\right]=\lim _{m \rightarrow \infty} \pi_{0}^{-1} \sum_{k=m+1}^{\infty} \theta^{-\alpha} \sigma_{t-k}^{\alpha} c_{k}^{\delta \alpha} .
$$

Hence (24) follows, which ends the proof of the theorem.

\section{Acknowledgment}

The authors would like to thank the referee for helpful comments that improved the paper. These results were obtained thanks to the support of AIRES-Sud, a programme from the French Ministry of Foreign and European Affairs implemented by the "Institut de Recherche pour le Développement (IRD-DSF)". The authors acknowledge grants from "Ministère de la Recherche Scientifique" of Senegal. 
A. Diop, S. Diouf, Journal Afrika Statistika, Vol. 5, Nº10, 2010, page 268-278.

\section{References}

[1] Ballerini, R. and McCormick, W.P., 1989. Extreme value theory for process with periodic variances. Comm. Statist. Stochastic Models . 5, 45-61.

[2] Coles, S., 2001. An Introduction to Statistical Modelling of Extreme Values. Springer, London.

[3] Davis, R.A. and Resnick, S.I., 1988. Extremes of moving average of random variables from the domain of attraction of the double exponential distribution. Stochastic Process. Appl. 30, 41-68.

[4] Davis, R.A. and Resnick, S.I., 1996. Limit theory for bilinear process with heavy-tailed noise. Ann. Appl. Probab. 6, 1191-1210.

[5] Eastoe, E.F. and Tawn, J.A., 2009. Modelling non-stationary extremes with to surface level ozone. J. R. Statist. Soc., Ser. C, Appl. Statist. 58, 25-45.

[6] Horowitz, J., 1980. Extreme values for nonstationary stochastic process: an application to air quality analysis. Technometrics. 22, 469-478.

[7] Kallenberg, O., 1983. Random measures, 3rd ed.Akademie, Berlin.

[8] Kulik, R., 2006. Limit theorem for moving average with random coefficients and heavy tailed noise. J. Appl. Probab. 43, 245-256.

[9] Neveu, J., 1976. Processus Ponctuels. Ecole d'Eté de Probabilités de Saint-Flour VI. Lecture Notes in Math., 598, Springer, New York.

[10] Niu, X-F., 1997. Extreme value theory for a class of nonstationary time series with applications. Ann. Appl. Prob. 7, 508-522.

[11] Resnick, S.I., 1986. Point processes, regular variation and weak convergence. Adv. in Appl. Probab. 18, 66-138.

[12] Resnick, S.I., 1987. Extreme values, Regular Variation and Point Process. Springer, New york. 